Artigo Original

\title{
| Alterações do nível pressórico e fatores de risco em graduandos de enfermagem*
}

\author{
Risk factors and alterations in blood pressure levels in undergraduate nursing students \\ Alteraciones del nível de la presión y factores de riesgo en profesionales graduandos en \\ enfermería
}

\section{Alba Lúcia Botura Leite de Barros ${ }^{1}$, Fabiana de Souza Vieira ${ }^{2}$, Cinthia Calsinski Assis ${ }^{3}$, Sandra Salloum Zeitoun ${ }^{4}$}

\section{RESUMO}

Objetivos: Identificar possíveis alterações do nível presóricoem graduandos de Enfermagem da Universidade Federal de São Paulo; Identificar fatores de risco para hipertensão arterial sistêmica; Verificar a associação entre os níveis pressóricos e os fatores de risco para hipertensão arterial sistêmica. Métodos: Estudo de corte transversal, desenvolvido entre novembro de 2006 a maio de 2007. A amostra foi composta por 120 graduandos de enfermagem da Universidade Federal de São Paulo. Foi aplicado um questionário específico sobre fatores de risco para hipertensão arterial e aferição da pressão arterial. O Teste de Fischer foi usado para determinar possíveis associações. Resultados: Dos graduandos, 92,5\% apresentaram níveis pressóricos normais, 4,2\% eram hipertensos e 3,3\% com pressão limítrofe. Notou-se o aparecimento de alguns fatores de risco para hipertensão arterial e doença cardiovascular. Conclusão: De acordo com os resultados, deve-se atentar para mudanças de hábito e estilo de vida mais saudável desses futuros enfermeiros.

Descritores: Hipertensão; Pressão arterial; Estudantes de enfermagem; Fatores de risco.

\begin{abstract}
Objectives: To identify risk factors and alterations in blood pressure levels in undergraduate nursing students and to examine the associations between the risk factors and blood pressure levels. Methods: A cross-sectional descriptive study was conducted between November 2006 and May 2007. The sample consisted of 120 undergraduate nursing students from the Federal University of São Paulo. A specific questionnaire on risk factors for high blood pressure was used. Blood pressure measures were taken and recorded. Fisher's exact test was used to determine associations between risk factors and high blood pressure. Results: The majority of participants (92.5\%) had normal blood pressure levels. A few students had high (4.2\%) or borderline (3.3\%) blood pressure levels. Students had some of the risk factors for high blood pressure and cardiovascular diseases. Conclusion: There is a need to emphasize lifestyle changes among those future nurses.
\end{abstract}

Keywords: Hypertension; Blood pression; Students, nursing; Risk factors.

\section{RESUMEN}

Objetivos: Medir la presión arterial para identificar posibles alteraciones en profesionales graduandos en Enfermería; identificar factores de riesgo para hipertensión arterial; verificar la asociación entre los niveles de presión y los factores de riesgo para hipertensión arterial. Métodos: Estudio de corte transversal, desarrollado entre noviembre de 2006 y mayo de 2007. La muestra fue compuesta por 120 graduandos de enfermería de la Universidad Federal de Sao Paulo. Fue aplicado un cuestionario específico sobre factores de riesgo para hipertensión arterial y para evaluar la presión arterial. La Prueba de Fischer fue usada para determinar posibles asociaciones. Resultados: 92,5\% de los graduandos presentaron niveles de presión normal, 4,2\% eran hipertensos y 3,3\% tenían presión limítrofe. Se notó el aparecimiento de algunos factores de riesgo para hipertensión arterial y enfermedad cardiovascular. Conclusión: De acuerdo con los resultados, se deben cambiar hábitos y estilo de vida de modo que sean más saludables para los futuros enfermeros.

Descriptores: Hipertensión; Presión sanguínea; Estudiantes de enfermería; Factores de riesgo.

\footnotetext{
* Trabalbo de Iniciação Científica (PIBIC/CNPQ), apresentado ao XV Congresso de Iniciação Científica da Universidade Federal de São Paulo- UNIFESP - São Paulo (SP), Brasil (2007).

${ }^{1}$ Professora Titular do Departamento de Enfermagem da Universidade Federal de São Paulo- UNIFESP - São Paulo (SP), Brasil.

${ }^{2}$ Acadêmica do $8^{a}$ semestre do Curso de Graduação em Enfermagem da Universidade Federal de São Paulo- UNIFESP - São Paulo (SP), Brasil.

${ }^{3}$ Pós-graduanda (Doutorado) em Enfermagem da Universidade Federal de São Paulo- UNIFESP - São Paulo (SP), Brasil.

${ }^{4}$ Doutora em Ciências. Professora do Programa de Pós-graduação da Faculdade de Ciências Médicas da Santa Casa de São Paulo FCMSCSP - São Paulo (SP), Brasil.
} 


\section{INTRODUÇÃO}

A hipertensão arterial sistêmica (HAS) é um dos principais fatores de risco para o desenvolvimento de acidente vascular encefálico, doença arterial coronariana, insuficiência cardíaca, insuficiência renal e doença arterial periférica, sendo responsável, nas últimas décadas, por um grande número de óbitos em todo país e uma das maiores causas de aposentadoria precoce permanente e a terceira causa de invalidez temporária, com conseqüente perda de anos de vida produtiva em adultos jovens. Portanto, é um importante fator de risco para as doenças cardiovasculares $(\mathrm{DCV})^{(1)}$.

A prevalência da HAS no mundo é estimada em $20 \%$ da população tornando as DCV uma das principais causas de morte no mundo ocidental. No Brasil, não há um estudo referente a toda a população, apenas estima-se a prevalência entre $22 \%$ e $44 \%$, com base em estudos realizados em algumas cidades: o estudo de Araraquara mostrou prevalência de $43 \%$ em 1990, em São Paulo no mesmo ano foi de $22 \%$, em Piracicaba em 1991 de 33\%, em Porto Alegre foi de 26\% em 1994, em Cotia foi de 44\% em 1997 e em Catanduva foi de $32 \%$ em $2000^{(2)}$.

Entre os fatores de risco conhecidos para HAS, encontram-se a idade, sexo, raça, história familiar, ingestão alimentar rica em gorduras, acréscimo de sal aos alimentos, obesidade, sedentarismo, estresse, tabagismo, ingestão de bebidas alcoólicas e uso de anticoncepcionais orais ${ }^{(3)}$. Também a relação cintura quadril (RCQ) pode revelar o risco de DCV, pois a localização abdominal de gordura associa-se, com grande freqüência, a condições tais como dislipidemias, resistência à insulina, diabetes e a $\mathrm{HAS}^{(3)}$.

Quanto maior a idade, maior é a prevalência de HAS, assim ela é mais comum na população idosa, cerca de $65 \%$ dos idosos são hipertensos ${ }^{(4)}$.Entretanto estudos apontam para a presença de fatores de risco para DCV já na fase adulta jovem ${ }^{(5)}$. Assim, optou-se em estudar uma população mais jovem, e em especial, os futuros profissionais da área de saúde.

\section{OBJETIVOS}

- Identificar possíveis alterações do nível pressórico dos graduandos de Enfermagem da Universidade Federal de São Paulo;

- Identificar fatores de risco para HAS;

- Verificar a associação entre os níveis pressóricos e os fatores de risco para HAS.

\section{MÉTODOS}

Trata-se de um estudo de corte transversal, desenvolvido após aprovação do Comitê de Ética em Pesquisa da UNIFESP. A coleta de dados foi realizada pela própria pesquisadora em um único encontro, previamente agendado, no período de novembro de 2006 a maio de 2007.
A seleção dos participantes atendeu aos seguintes critérios de inclusão: ser graduando de enfermagem da UNIFESP com idade igual ou superior a 18 anos, ter assinado o Termo de Consentimento Livre e Esclarecido.

A população foi constituída por 331 graduandos de enfermagem, sendo 90 matriculados na $1^{\text {a }}$ série, 86 na $2^{\mathrm{a}}$ série, $85 \mathrm{na} 3^{\text {a }}$ série e $70 \mathrm{na} 4^{\mathrm{a}}$ série. Deste total, foi realizado um processo de amostragem estratificada por série e subestrato por sexo. Foram selecionados todos os alunos do sub-estrato do sexo masculino e realizada a seleção do subestrato do sexo feminino de forma aleatória, por sorteio.

O tamanho da amostra foi calculado com base em uma amostragem estratificada com partilha proporcional. Assim, foi verificada a necessidade de avaliação de 120 indivíduos distribuídos nos estratos ${ }^{(6)}$ (Tabela 1 ).

Estudos sobre a prevalência de HAS têm divergido quanto ao número de aferições da pressão arterial (PA) utilizadas para classificar o indivíduo como hipertenso, variando desde duas aferições ${ }^{(-.9)}$ até seis aferições ${ }^{(10)}$. Neste estudo, optou-se por realizar duas medidas da PA como sugerido pelo VII Joint National Comittee ${ }^{(11)}$, ou seja, uma ao início e outra ao término da aplicação de questionário específico sobre fatores de risco para HAS formulado por Simão(12). Em seguida, foi obtida a média das duas. Os entrevistados foram classificados como hipertensos utilizando o critério proposto pela $\mathrm{V}$ Diretriz Brasileira de Hipertensão, apresentado, a seguir, na Quadro 1, onde HAS é definida como pressão arterial sistólica $(\mathrm{PAS}) \geq 140 \mathrm{mmHg}$ e/ou pressão arterial diastólica

Tabela 1 - Número de alunos segundo sexo, por série da graduação de enfermagem. São Paulo, 2007.

\begin{tabular}{cccc}
\hline Série & Feminino & Masculino & Total \\
\hline $1^{\mathrm{o}}$ & 28 & 4 & 32 \\
$2^{\mathrm{o}}$ & 27 & 5 & 32 \\
$3^{\mathrm{o}}$ & 25 & 7 & 32 \\
$4^{\mathrm{o}}$ & 21 & 3 & 24 \\
\hline Total & 101 & 19 & 120 \\
\hline
\end{tabular}

Quadro 1 - Classificação diagnóstica dos níveis pressóricos de acordo com a V Diretriz Brasileira de Hipertensão Arterial de 2006. São Paulo, 2007.

\begin{tabular}{|c|c|c|}
\hline Classificação da PA & $\begin{array}{c}\text { PAS* } \\
(\mathrm{mmHg})\end{array}$ & $\begin{array}{c}\text { PAD* }^{*} \\
(\mathrm{mmHg})\end{array}$ \\
\hline Ótima & $<120$ & $<80$ \\
\hline Normal & $<130$ & $<85$ \\
\hline Limítrofe & $130-139$ & $85-89$ \\
\hline \multicolumn{3}{|l|}{ Hipertensão } \\
\hline Estágio 1 (Leve) & 140-159 & $90-99$ \\
\hline Estágio 2 (Moderado) & $160-179$ & $100-109$ \\
\hline Estágio 3 (Grave) & $>=180$ & $>=110$ \\
\hline - Sistólica Isolada & $>=140$ & $>=90$ \\
\hline
\end{tabular}


(PAD) $\leq 90 \mathrm{mmHg}$, ou, quando for o caso, a observação indivíduo em uso de medicação anti-hipertensiva.

\section{Observações}

O método utilizado para a medida da PA foi o indireto, com técnica auscultatória. Foi utilizado esfigmomanômetro do tipo coluna de mercúrio previamente testado e devidamente calibrado.

O preparo do entrevistado para realizar a medida da PA seguiu as normas da V Diretriz Brasileira de Hipertensão Arterial ${ }^{(13)}$ :

- Explicar o procedimento. Informar a necessidade de realizar repouso de cinco minutos em ambiente calmo; evitar bexiga cheia; não praticar exercícios físicos 60 a 90 minutos antes; não ingerir bebidas alcoólicas, café ou alimentos e não fumar 30 minutos antes; manter pernas descruzadas no momento da aferição, posicionar o braço na altura do coração com a palma da mão voltada para cima e cotovelo ligeiramente fletido.

- Medir a circunferência do braço e selecionar o manguito de tamanho adequado ao braço. Colocar o manguito sem deixar folgas acima da fossa cubital, cerca de dois a três $\mathrm{cm}$; centralizar o meio da parte compressiva do manguito sobre a artéria braquial; estimar o nível da pressão sistólica (palpar o pulso radial e inflar o manguito até seu desaparecimento, desinflar rapidamente e aguardar um minuto antes da medida); palpar a artéria braquial na fossa cubital e colocar a campânula do estetoscópio sem compressão excessiva; inflar rapidamente até ultrapassar 20 a $30 \mathrm{mmHg}$ o nível estimado da pressão sistólica; proceder à deflação lentamente; determinar a pressão sistólica na ausculta do primeiro som (fase I de Korotkoff), que é um som fraco, seguido de batidas regulares; determinar a pressão diastólica no desaparecimento do som (fase V Korotkoff); informar os valores de pressão arterial.

Logo após, foram mensurados os dados antropométricos: peso, altura, medida da cintura e quadril.

A medida da cintura foi obtida pelo valor de seu perímetro no ponto médio da menor curvatura entre a costela e a crista ilíaca utilizando uma fita métrica ${ }^{(12-13)}$.

A medida do quadril foi obtida pelo valor de seu perímetro na sua área de maior protuberância, utilizando uma fita métrica ${ }^{(12)}$.

Para avaliação do estado nutricional, foi calculado o Índice de Massa Corporal (IMC) e adotada a classificação preconizada pela Organização Mundial de Saúde que considera baixo peso IMC menor $18,5 \mathrm{Kg} / \mathrm{m}^{2}$; peso normal de 18,5 e $24,9 \mathrm{Kg} / \mathrm{m}^{2}$; pré-obesidade de 25 a $29,9 \mathrm{Kg} / \mathrm{m}^{2}$ e obesidade maior ou igual a $30 \mathrm{Kg} / \mathrm{m}^{2:}$ nível I de 30 a $34,9 \mathrm{Kg} /$ $\mathrm{m}^{2}$; nível II de 35 a $39,9 \mathrm{Kg} / \mathrm{m}^{2}$ e nível III maior ou igual 40 $\mathrm{Kg} / \mathrm{m}^{2(12-13)}$.

$\mathrm{Na}$ medida da RCQ, consideraram-se os pontos de corte adequados para as mulheres quando obtidos valores menores ou iguais a 0,85 e para os homens menores ou iguais a $0,95^{(12-}$ 13).

$\mathrm{Na}$ medida da cintura, o perímetro acima de $80 \mathrm{~cm}$ para as mulheres e acima de $94 \mathrm{~cm}$ para os homens foi considerado um risco aumentado e quando o valor foi maior ou igual a 88 $\mathrm{cm}$ para as mulheres e maior ou igual a $102 \mathrm{~cm}$ para os homens o risco tornou-se muito mais elevado ${ }^{(12)}$.

Para verificar a associação entre os níveis pressóricos e os fatores de risco para HA foi utilizado o Teste Exato de Fisher para a realização do cálculo. O nível de significância adotado foi de $5 \%$.

\section{RESULTADOS}

Participaram do estudo 120 graduandos de enfermagem, sendo $9(15,8 \%)$ do sexo masculino e $101(84,2 \%)$ do sexo feminino. A média de idade foi de 21,6 e desvio padrão de 3,3. A grande maioria, $91(75,8 \%)$ referiu ser de cor branca, $16(13,4 \%)$ pardos, 9 (7,5\%) amarelos e $4(3,3 \%)$ negros.

Apresentaram alteração do nível pressórico $9(7,5 \%)$ dos alunos, Assim, foi verificada PAS maior ou igual a $140 \mathrm{mmHg}$ e/ou PAD maior ou igual a $90 \mathrm{mmHg}$ em quatro alunos e somente um declarou ser hipertenso, em uso de medicação anti-hipertensiva, portanto $5(4,2 \%)$ dos estudantes apresentaram HA e 4 (3,3\%) apresentaram pressão limítrofe.

A média da PAS foi de $114 \mathrm{mmHg}$ e a PAD foi de 74,6 $\mathrm{mmHg}$.

Em relação aos fatores de risco, $16(13,3 \%)$ estudantes apresentavam peso acima do recomendado, como pode ser visto na Figura 1.

A grande maioria dos estudantes de enfermagem (92,5\%) apresentou uma RCQ dentro dos padrões normais, segundo os dados apresentados na Figura 2.

Foi também analisada a circunferência abdominal de forma isolada, já que ela, por si só, constitui um fator de risco para as complicações metabólicas e DCV (Figura3).

Em relação às histórias familiares de $\mathrm{HA}$, os alunos referiram que 60 (50\%) dos pais, nove $(7,5 \%)$ dos irmãos, 80 $(66,7 \%)$ dos avós e $65(54,2 \%)$ dos tios dos alunos eram hipertensos.

A realização de atividades físicas está relacionada com um estilo de vida saudável, e foi constatado que $65(54,2 \%)$ praticavam algum tipo de atividade física, e destes, sete $(10,8 \%)$ que realizavam caminhada, o faziam de modo não programado, ou seja, consideraram caminhada as atividades diárias como o deslocamento da universidade para casa e vice-versa.

Em relação ao uso do tabaco, 119 (99,2\%) negaram seu uso. Por outro lado, o consumo de bebida alcoólica foi significativo em $41(34,2 \%)$ dos alunos.

O padrão alimentar foi interrogado com base na classificação dos grupos nutricionais (Figura 4).

Além disto, constatou-se que $31(25,8 \%)$ dos alunos têm o hábito de ingerir alimentos preparados com excesso sal. Em relação ao nível de estresse, 58 (48,3\%) dos graduandos consideraram-se pessoas estressadas.

As variáveis sexo, IMC, medida da cintura e consumo de gordura no jantar apresentaram associação com a elevação dos níveis pressóricos (pressão limítrofe ou hipertensão). 


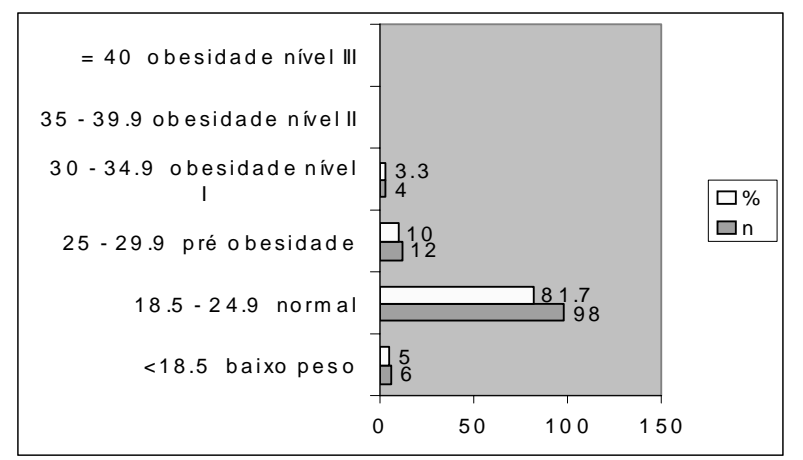

Figura 1. Graduandos de enfermagem, segundo o índice de massa corporal $\left(\mathrm{Kg} / \mathrm{m}^{2}\right)$ de acordo com a classificação da Organização Mundial de Saúde. São Paulo, 2007.

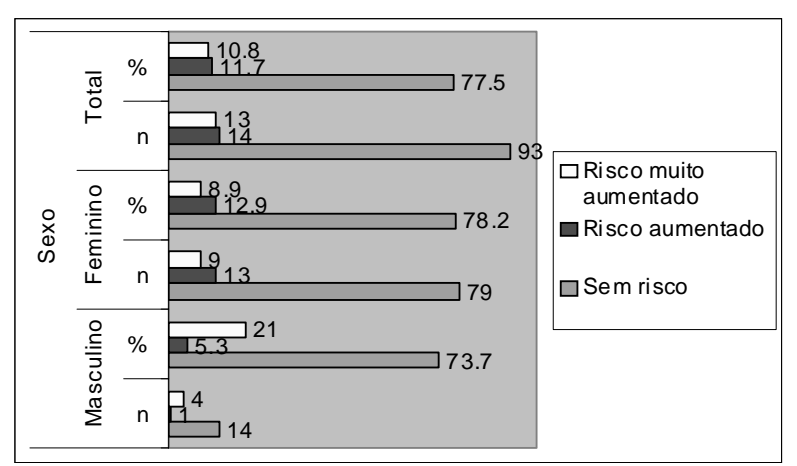

Figura 3. Graduandos de enfermagem segundo a classificação de risco para Doenças Cardiovasculares com base na circunferência abdominal e sexo. São Paulo, 2007.

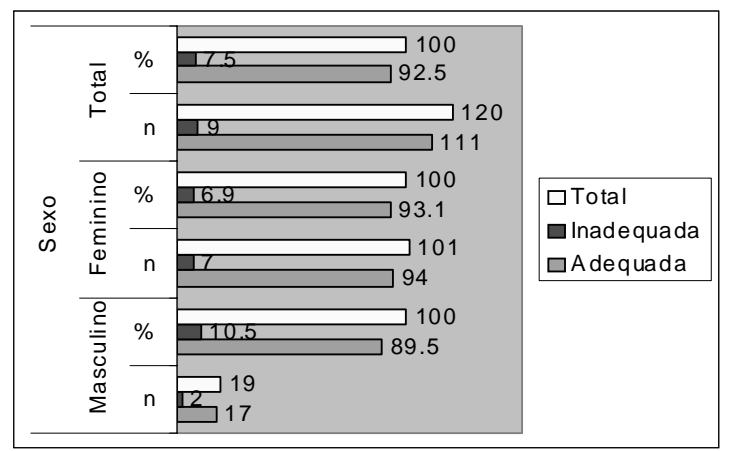

Figura 2. Graduandos de enfermagem, segundo a relação cintura/quadril com pontos de corte propostos pela V Diretriz Brasileira de Hipertensão de 2006 e sexo. São Paulo, 2007

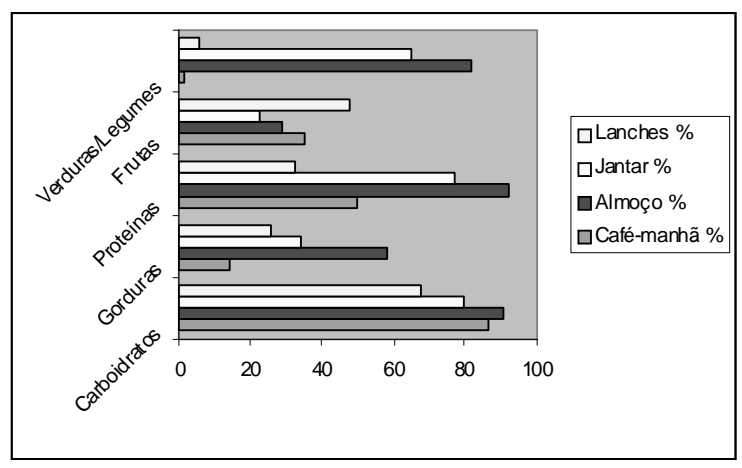

Figura 4. Graduandos de enfermagem, segundo o habito de ingestão dos grupos nutricionais nas refeições. São Paulo, 2007.

Tabela 2. Graduandos de enfermagem, segundo as variáveis que obtiveram associação e os níveis pressóricos.São Paulo, 2007.

\begin{tabular}{|c|c|c|c|c|c|c|c|}
\hline \multirow{2}{*}{ Variáveis } & \multicolumn{2}{|c|}{ Pressão normal } & \multicolumn{2}{|c|}{ Pressão limítrofe } & \multicolumn{2}{|c|}{ Hipertensão } & \multirow[t]{2}{*}{ Valor de $\mathrm{p}^{*}$} \\
\hline & $\mathrm{n}$ & $\%$ & $\mathrm{n}$ & $\%$ & $\mathrm{n}$ & $\%$ & \\
\hline \multicolumn{8}{|l|}{ Sexo } \\
\hline Masculino & 13 & 11,7 & 3 & 75,0 & 3 & 60,0 & \multirow{2}{*}{0,0006} \\
\hline Feminino & 98 & 88,2 & 1 & 25.0 & 2 & 40,0 & \\
\hline \multicolumn{8}{|l|}{ IMC } \\
\hline Baixo peso & 5 & 4,5 & 0 & 0,0 & 1 & 20,0 & \multirow{4}{*}{$<0,0001$} \\
\hline Normal & 96 & 86,4 & 1 & 25,0 & 1 & 20,0 & \\
\hline Pré-obesidade & 8 & 7,2 & 3 & 75,0 & 1 & 20,0 & \\
\hline Obesidade nível I & 2 & 1,8 & 0 & 0,0 & 2 & 40,0 & \\
\hline \multicolumn{8}{|l|}{ Medida da cintura } \\
\hline Normal & 89 & 80,1 & 2 & 50,0 & 2 & 40,0 & \multirow[t]{2}{*}{0,0409} \\
\hline Elevada & 22 & 19,8 & 2 & 50,0 & 3 & 60,0 & \\
\hline \multicolumn{8}{|c|}{ Consumo de gordura no jantar } \\
\hline Sim & 37 & 33,3 & 0 & 0,0 & 4 & 80,0 & \multirow{2}{*}{0,0087} \\
\hline Não & 74 & 66,6 & 4 & 100,0 & 1 & 20,0 & \\
\hline
\end{tabular}

Legenda: "p-valor: baseado no Teste Exato de Fisher. 
Assim a pressão arterial limítrofe foi mais freqüente, entre alunos do sexo masculino e, entre aqueles com pré obesidade e obesidade nível I (Tabela 1).

\section{DISCUSSÃO}

A baixa prevalência de HA $(4,2 \%)$ encontrada no presente estudo reflete os achados da literatura, considerando que a grande maioria da população estudada é jovem.

Há poucos estudos específicos sobre HA na população adulta jovem, mas há dois estudos que, apesar de serem antigos, mostram-se completos: o estudo realizado com a população de Volta Redonda-Rio de Janeiro, que utilizou o critério adotado pela OMS, na ocasião com pontos de corte de PA de 160/95 mmHg, evidenciou uma prevalência baixa de HA $(2,9 \%)^{(14)}$, e o outro ${ }^{(5)}$, realizado com estudantes entre 16 e 25 anos de idade na cidade de Botucatu-São Paulo, com pontos de corte de PA de $140 / 90 \mathrm{mmHg}$, que evidenciou uma prevalência de $5,04 \%$.

Em um estudo realizado na cidade de Fortaleza-CE, no período de 2004 a 2007, objetivando comparar dois grupos de escolares, onde o grupo-caso foi representado por estudantes com alteração da PA e o grupo-controle sem alteração da mesma mostrou, entre outros achados, que nos dois grupos foram encontrados fatores de risco para a HAS, sendo as medidas antropométricas medianamente superiores no grupocaso e o fumo passivo mais presente no grupo-controle ${ }^{(15)}$.

A obesidade é um fator de risco para HAS, podendo ser responsável por cerca de $20 \%$ a $30 \%$ dos casos, e $75 \%$ dos homens e $65 \%$ das mulheres apresentam HAS diretamente atribuída ao sobrepeso e obesidade ${ }^{(13)}$. Neste estudo, $40 \%$ dos considerados hipertensos possuem obesidade grau I, enquanto este número se reduz para $2 \%$ quando este não é obeso.

Em um estudo realizado no Ambulatório de Obesidade da UNIFESP de 1998 a 1999 foi verificada a influência do IMC sobre a prevalência de HAS, sendo observada a relação entre o aumento da incidência de hipertensão e elevação do IMC, pois eram hipertensos $23 \%$ dos pré-obesos; $32,7 \%$ com obesidade nível I; 55\% com obesidade nível II e 67,1 \% com obesidade nível IIII ${ }^{(16)}$.

A localização abdominal de gordura tem grande impacto sobre as DCV por associar-se com grande frequência com dislipidemias, diabetes e a HAS que favorecem a eventos cardiovasculares ${ }^{(13)}$. Independentemente do sobrepeso, a gordura abdominal é um importante fator de risco para essas condições $^{(13)}$.

A obesidade central está fortemente relacionada aos níveis de PA, ou seja, indivíduos com nível de pressão arterial ótimo que no decorrer do tempo, apresentam obesidade central têm maior incidência de $\mathrm{HAS}^{(13)}$.

Neste estudo, foi observada a associação entre a medida da cintura e a elevação dos níveis tensionais, sendo que $60 \%$ dos indivíduos hipertensos e $50 \%$ daqueles com pressões limítrofes possuíam aumento da circunferência da cintura em comparação com os $20 \%$ sem alteração da PA.

$O$ resultado de antecedentes familiares de HAS evidenciou o quão prevalente é esta doença na sociedade, mostrando seu real custo financeiro à sociedade com frequentes internações e aposentadorias precoces ou afastamento do trabalho. Em 2005 ocorreram 1.180.184 internações por $\mathrm{DCV}$, custo global de $\mathrm{R} \$ 1.323 .775 .008,28^{(13)}$. Estes resultados se assemelham aos encontrados em outros estudos, onde aproximadamente $40 \%$ de um dos pais apresentavam $\mathrm{HAS}^{(12)}$.

Há um grande número de alunos que se consideram pessoas estressadas (48,3\%). Estudos já realizados demonstram que o período de maior ansiedade ocorre no início dos estágios curriculares, assim, a vida acadêmica contribui para este perfil psíquico ${ }^{(17)}$. Em um estudo realizado em 2000 com estudantes de uma universidade do Rio de Janeiro, evidenciou-se que $50 \%$ dos alunos se consideraram estressados, $40,9 \%$ se irritavam facilmente e $63,6 \%$ sentiamse fatigados ${ }^{(18)}$. Estudos experimentais demonstram elevação transitória de PA em situações de estresse, como o estresse mental. Estudos mais recentes evidenciam o efeito do estresse psicoemocional na reatividade cardiovascular e na PA, podendo contribuir para a HAS sustentada ${ }^{(13)}$.

Sabendo que o sedentarismo aumenta a incidência de HAS, indivíduos sedentários apresentam risco 30\% maior de desenvolver HAS que os ativos ${ }^{(13)}$.

O estudo demonstrou um elevado número de alunos sedentários (45,8\%). Na literatura, encontraram-se resultados que variam de $13,8 \%^{(12)}$ a $74,5 \%$ de universitários sedentários em um Departamento de Enfermagem, sendo os participantes a grande maioria constituída por alunos ${ }^{(19)}$.

O consumo elevado de bebidas alcoólicas como cerveja, vinho e destilados aumenta a $\mathrm{PA}$, segundo a literatura. $\mathrm{O}$ efeito varia com o sexo, e a magnitude está associada à quantidade de etanol e à frequência de ingestão. $O$ efeito do consumo leve a moderado de etanol não está definitivamente estabelecido. Verifica-se redução média de $3,3 \mathrm{mmHg}$ na PAS e $2 \mathrm{mmHg}$ na PAD com a redução do consumo de $\operatorname{etanol}^{(13)}$.

Em relação ao padrão alimentar adotado pelos alunos foi favorável à prevenção de HAS, já que evidenciou consumo moderado de frutas, verduras e legumes. A dieta preconizada pelo estudo DASH (Dietary Approachs to Stop Hypertension) mostrou benefícios no controle da PA, inclusive em pacientes fazendo uso de anti-hipertensivos, enfatizando o consumo de frutas, verduras, alimentos integrais, leite desnatado, quantidade reduzida de gorduras saturadas e colesterol ${ }^{(13)}$. Apenas o consumo de gorduras no jantar obteve uma associação com a HAS e, portanto, favorável ao aparecimento da mesma.

O consumo excessivo de sal pelos alunos é um dado preocupante, já que a relação entre o aumento da PA e avanço da idade é maior em populações com alta ingestão de sal ${ }^{(13)}$.

\section{CONCLUSÃO}

A maioria dos alunos de enfermagem da UNIFESP percentil pequeno de alterações do nível pressórico: $4,2 \%$ de 
hipertensos e 3,3\% de pressão limítrofe, porém, notou-se o aparecimento de alguns fatores de risco para HAS e DCV. Sendo assim, deve-se atentar para medidas que promovam mudanças de hábitos e estilo de vida mais saudável, pensando nos anos seguintes e na qualidade da vida dos futuros profissionais da área da saúde.

Dentre os fatores de risco para HA, houve associação com a elevação dos níveis tensionais as variáveis sexo, IMC, cintura e consumo de gordura no jantar, assim é necessário a promoção de hábitos alimentares mais saudáveis para controlar os níveis pressóricos dos alunos com HA e pressão limítrofe, prevenindo, assim, a doença nos alunos com presença de fatores de risco, mas sem alteração dos níveis pressóricos.

\section{Agradecimentos}

Os autores agradecem ao CNPq pelo apoio financeiro.

\section{REFERÊNCIAS}

1. Souza NRM, Souza e Silva NA. Trabalho e hipertensão arterial. A responsabilidade social das empresas: problemas, oportunidades e possíveis estratégias de intervenção. Rev SOCERJ. 2003;16(1):60-4.

2. IV Diretrizes Brasileiras de Hipertensão Arterial. Arq Bras Cardiol. 2004; 82 (Supl 4): 1-14.

3. Ferreira MG, Valente JG, Gonçalves-Silva RMV, Sichieri R. Acurácia da circunferência da cintura e da relação cintura/quadril como preditores de dislipidemias em estudo transversal de doadores de sangue de Cuiabá, Mato Grosso, Brasil. Cad Saúde Pública. 2006;22(2):307-14.

4. Freitas EV, Brandão AP, Brandão AA, Magalhães ME, Pozzan R, Zilli E, Pozzan R. Peculiaridades na abordagem do idoso hipertenso. Rev SOCERJ. 2002;15(4):256-62.

5. Almeida DB, Habermann F, Soares VA, Monteiro Filho RC, Ferreira ES, Abujamra Júnior O, et al. Estudo comparativo da pressão arterial e da prevalência da hipertensão arterial em duas coortes sucessivas (19751976) de estudantes de 16 a 25 anos, Botucatu, SP, Brasil. Rev Saúde Pública. 1978;12(4):497-505.

6. Cochran WG. Sampling techniques. $3^{\text {rd }}$ ed. New York: John Wiley \& Sons; 1977.

7. Lolio CA. Prevalência de hipertensão arterial em Araraquara. Arq Bras Cardiol. 1990;55 (3):167-73.

8. Gus I, Harzheim E, Zaslavsky C, Medina C, Gus M. Prevalência, reconhecimento e controle da hipertensão arterial sistêmica no estado do Rio Grande do Sul. Arq Bras Cardiol. 2004;83(5):424-33.

9. Castro RAA. Prevalência de hipertensão arterial sistêmica na cidade de Formiga, MG [dissertação]. São Paulo: Universidade Federal de São Paulo, Escola Paulista de Medicina; 2005.

10. Burt VL, Whelton P, Roccella EJ, Brown C, Cutler JA, Higgins $\mathrm{M}$, et al. Prevalence of hypertension in the US adult population. Results from the Third National Health and Nutrition Examination Survey, 1988-1991. Hypertension. 1995;25(3):305-13.

11. The Seventh Report of the Joint National Committee on Prevention, Detection, Evaluation, and Treatment of High Blood Pressure (JNC 7) [Internet]. Bethesda (MD): National Institutes of Health-National Heart, Lung, and Blood Institute (US); 2007. [citado 2007 Maio 15]. Disponível em: http://www.nhlbi.nih.gov/ guidelines/index.htm.

12. Simão M. Hipertensão arterial e fatores de risco associados: estudo entre universitários da cidade de Lubango-Angola [tese]. Ribeirão Preto: Escola de Enfermagem de Ribeirão Preto da Universidade de São Paulo; 2005.

13. Sociedade Brasileira de Hipertensão. V Diretrizes Brasileiras de Hipertensão Arterial [Internet]. [citado 2006 Nov 27]. Disponível em: http://www.sbn.org.br/ Diretrizes/V_Diretrizes_Brasileiras_de_Hipertensao_ Arterial.pdf

14. Klein $\mathrm{CH}$, Araújo JWC, Leal MC. Inquérito sobre hipertensão arterial em Volta Redonda - RJ. Cad Saúde Publica. 1985;1(1):58-70.

15. Araújo TL, Lopes MVO, Oliveira ARS, Chaves DBR, Costa AGS, Alves FEC, et al. Fatores de risco para hipertensão arterial em escolares: um estudo de casocontrole. Rev Enferm UERJ. 2008;16(2):149-55.

16. Carneiro G, Faria AN, Ribeiro Filho FF, Guimarães A, Lerário D, Ferreira SRG, et al. Influência da distribuição da gordura corporal sobre a prevalência de hipertensão arterial e outros fatores de risco cardiovascular em indivíduos obesos. Rev Assoc Med Bras. 2003;49(3):306-11.

17. Farah OGD. Stress e coping no estudante de graduação de enfermagem: identificação e atuação [tese]. São Paulo: Escola de Enfermagem da Universidade de São Paulo; 2001.

18. Mauro MYC, Santos CC, Oliveira MM, Lima PT. O estresse e a prática de enfermagem: quando parar e refletir? Uma experiência com estudantes de enfermagem. Acta Paul Enferm. 2000;13(N Esp Pt 2):44-8.

19. Rosa SCD. Medida comparativa da pressão arterial no braço e antebraço [dissertação]. Guarulhos (SP): Universidade de Guarulhos; 2006. 